\title{
Maximization of wave power extraction of a heave point absorber with a
} sea-state-based causal control algorithm

\author{
Liang $\mathrm{Li}^{*}$, Zhen Gao \\ *Corresponding author: liang.li@strath.ac.uk.
}

\begin{abstract}
A causal control strategy is developed to tackle the non-causality arising in the real-time implementation of optimal wave energy control. The proposed control strategy utilizes a causal approximation transfer function to link the current wave force to the desired buoy velocity so as to eliminate the non-causality. An optimum model of the approximation transfer function is derived considering the power density distribution of the local wave spectrum. Based on the optimized approximation transfer function, the desired buoy velocity is achieved by tuning the power take-off mechanical force with the PID control. The efficiency of the proposed causal control strategy is assessed for a heaving point-absorber, in a set of random wave conditions. Generally, the heaving point-absorber could extract wave power up to $90 \%$ of the theoretical upper bound using the proposed control strategy. The sensitivity of the proposed control to viscous damping effect due to drag and wave spectrum bandwidth is investigated. Although it is less efficient in broad-banded sea state, the control efficiency is still within an acceptable level (above 70\%).
\end{abstract}

Keywords: wave energy converter; wave power extraction; complex-conjugate control; non-causality; transfer function; real-time implementation.

\section{Introduction}

Given the advantages of high power density, wave energy is regarded as a prospective solution to the sustainable generation of power. Device used to harvest energy from ocean waves is called wave energy converter (WEC). Among various categories of WECs, the oscillating-body WEC may be the most common which converts the kinetic energy of the buoy under wave loads into electric power. A significant drawback of the oscillating-body WEC is that the bandwidth of energy capture is relatively narrow so that the performance outside the resonance frequency range is poor. Therefore, the energy extraction efficiency in random waves is unsatisfactory. Although the multi-stable mechanism has been recently adopted $[1,2]$, real-time control is still the primary approach to increase energy conversion efficiency.

Wave energy control theory in the early stage was developed in the frequency domain. Falnes [3] presented a detailed interpretation of the complex-conjugate control, which maximizes the energy harvesting by changing the mechanical force of the power take-off (PTO) system. Budal and Falnes [4] proposed the latching control, which makes the velocity in phase with the wave excitation force by 
1 locking the buoy at some time instants. Other control algorithms include declutching control [5], passive 2 phased control [2, 6], harmonic balance control [7], moment-matching control [8], pseudo-spectral control [9], etc. The application of wave control has been proved successful in regular wave [10]. However, the real-time implementation of optimal wave energy control in random waves is still a significant challenge due to the non-causality as the transformation from the frequency domain into the time domain results in a non-causal transfer function. More specifically, the current control command of optimal wave energy control depends on future wave excitation forces. Despite the full development of optimal wave energy control theory, the non-causality issue is not addressed sufficiently, and many previous researchers neglected it in their real-time implementation of optimal control. For instance, Henriques et al. [11] assumed that the future wave force was known when they applied the real-time latching control to an oscillating water column WEC.

Considering that optimal wave energy control depends on future wave excitation forces, many researchers aim to tackle the non-causality directly by predicting future wave forces. Fusco and Ringwood [12] developed a linear auto-aggressive model to predict short-term future wave forces. $\mathrm{Li}$ et al. [13] trained the artificial neural network for the coming wave force forecasting. Wang et al. [14] gave a critical review of machine learning for forecasting application. Other forecasting approaches include the grey model [15] and spectral method [16]. The application of wave force forecasting in optimal wave energy control is still facing enormous challenges. First, it is difficult to guarantee the forecasting accuracy. As shown by Li et al. [17], the accuracy of the forecasting has a significant influence on the control performance and the energy harvesting is hardly increased in the case of high forecasting error. Also, the wave direction changes frequently in real conditions and the WEC may be located in short-crested waves, making the wave force forecasting even more difficult.

This paper presents an alternative solution to the non-causality by proposing a sub-optimal causal transfer function to substitute the optimal non-causal transfer function, which links the wave excitation force to the desired WEC oscillation velocity. Although the proposed control is sub-optimal, it becomes possible to be applied in real conditions as the non-causality is eliminated. Similar ideas have been documented previously. Fusco and Ringwood [18] used a causal frequency domain transfer function in the real-time implementation of wave energy control by assuming that the random wave forces are sinusoidal. It indicates that the power extraction is only maximized for a single frequency. The sinusoidal assumption was also adopted by Shek et al. [19] and Wu et al. [20]. These methods all assumed that the wave forces were harmonic and maximized the power extraction at a single critical frequency (the peak period of the random waves). Nevertheless, the ocean waves are polychromatic, and the transfer function is not optimized for other frequency components.

In the present research, a simplified but effective approach to tackle non-causality is proposed, which replaces the optimal transfer function with a causal and sub-optimal approximation substitution. Based on the local wave spectrum, an optimum model is developed to optimize the approximation transfer 
1 function. The optimum model takes account of the power density distribution of the wave spectrum so

2 that it is more efficient than those optimized for an individual frequency. The optimized approximation

3 transfer function is then used to estimate the desired buoy velocity, which will be obtained using the

4 PID control. The efficiency and the robustness of the proposed control strategy are examined in a variety

5 of random waves.

\section{2. Numerical model}

Fig. 1 gives the sketch of the heaving point-absorber WEC studied in this work. The point-absorber consists of a buoy harvesting wave energy and a PTO system converting wave energy into electrical power. The buoy, a hemisphere with a radius of $2.5 \mathrm{~m}$, is rigidly connected to the PTO system fixed at the seabed. A right-handed coordinate system attached to the earth is used (see Fig. 1). The centre of the coordinate system is fixed at the mean sea surface. $Z$ axial is positive upward. $X$ axial is along the propagation direction of the sea waves. Only heave motion of the buoy is allowed.

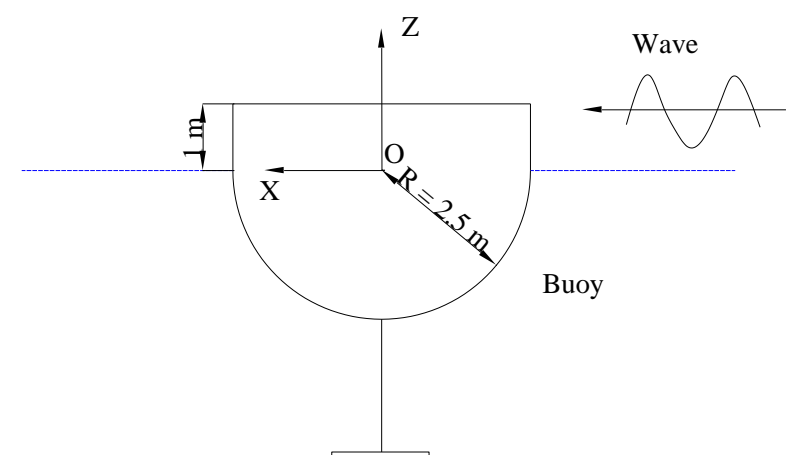

PTO system

Fig. 1. Wave energy converter.

The linear potential flow theory is adopted to address the wave-structure interaction. Based on the impulse response theory [21], the time-domain motion equation of the buoy is given by

$$
(M+m) \ddot{z}(t)+\int_{0}^{t} \kappa(t-\tau) \dot{z}(\tau) \mathrm{d} \tau+D \dot{z}(t)+\rho g \pi R^{2} z(t)=f_{\text {wave }}(t)+f_{\text {PTO }}(t)
$$

where $M=33543 \mathrm{~kg}$ is the mass of the buoy and $m=16410 \mathrm{~kg}$ is the added mass at infinite frequency. $z, \dot{z}$, and $\ddot{z}$ are the displacement, the velocity, and the acceleration. Since the potential flow theory can not take the viscous damping into account, an extra damping coefficient $D$ is added to represent the drag force. In accordance with the developed control strategy (please refer to Section 3), a linear viscous 
1 damping model is adopted. In the present simulation, $D=0.01 \times 2 \sqrt{\rho g \pi R^{2}(M+m)}$ is used ( $1 \%$ critical

2 damping). Please note that $1 \%$ critical damping may not represent the real situation so that a sensitivity 3 study on the viscous damping is conducted in Section 5.2 where it will be shown that the proposed control algorithm is effective regardless of the viscous damping. $\rho$ is the water density. $g$ is the gravity acceleration. $f_{\text {wave }}$ is the wave excitation force, and $f_{\text {РTO }}$ is the PTO mechanical force acting on the buoy. $\kappa$ is the so-called retardation kernel function which represents the memory effect of wave radiation force

Although Eq. (1) is widely used to simulate the wave-structure interaction, such form makes it inconvenient to implement the control strategy. An alternative model is thus developed to simulate the dynamics of the point-absorber in random waves, in which the convolution term is replaced by a statespace representation.

$$
\begin{aligned}
& \dot{\boldsymbol{u}}(t)=\boldsymbol{A} \cdot \boldsymbol{u}(t)+\boldsymbol{B} \dot{z}(t) \\
& f(t)=\boldsymbol{C} \cdot \boldsymbol{u}(t)
\end{aligned}
$$

where $n$ is the order of ordinary differential equation Eq. (2). $\boldsymbol{u}(t)$ is a state vector with dimension $n \times 1$, which can be regarded as an intermediate variable. $\boldsymbol{A}, \boldsymbol{B}$ and $\boldsymbol{C}$ are matrices with dimensions $n \times n, n \times 1$ and $1 \times n$, the values of which are given in Eq. (3).

$$
\begin{aligned}
\boldsymbol{A} & =\left[\begin{array}{cccc}
-2.932 & -6.302 & -4.927 & -2.581 \\
1 & 0 & 0 & 0 \\
0 & 1 & 0 & 0 \\
0 & 0 & 1 & 0
\end{array}\right] \\
\boldsymbol{C} & =\left[\begin{array}{lll}
31728 & 4489529824 & 0
\end{array}\right]
\end{aligned}
$$

Define a state vector $\boldsymbol{x}(t)=\left[z(t), \dot{z}(t), \boldsymbol{u}(t)^{T}\right]^{T}$ with dimension $(n+2) \times 1$. Then Eq. (1) is reexpressed as

$$
\begin{aligned}
& \dot{\boldsymbol{x}}(t)=\boldsymbol{\phi} \cdot \boldsymbol{x}(t)+\boldsymbol{\varphi} \\
& \boldsymbol{\phi}=\left[\begin{array}{ccc}
0 & 1 & \mathbf{0} \\
-\frac{\rho g \pi R^{2}}{M+m} & -\frac{D}{M+m} & -\frac{\boldsymbol{C}}{M+m} \\
\mathbf{0} & \boldsymbol{B} & \boldsymbol{A}
\end{array}\right], \boldsymbol{\varphi}=\left[\begin{array}{c}
0 \\
\frac{f_{\text {wave }}(t)+f_{P T O}(t)}{M+m} \\
\mathbf{0}
\end{array}\right]
\end{aligned}
$$

Please refer to [22] for more details regarding the development of the state-space model. Eq. (4) is a first-order differential formula and is solved using the 4-th order Runge-Kutta method. Then, the average power extraction is given by

$$
P=\frac{1}{T} \int_{0}^{T}-f_{P T O}(t) \cdot \dot{z}(t) \mathrm{d} t
$$

Please note that Eq. (5) represents the hydrodynamic power absorption. If the final electrical power is to be investigated, a wave-to-wire model [23] should be employed.

All the frequency-domain hydrodynamic coefficients (added mass, radiation damping, and wave force transfer function) used to derive the state-space model are calculated with the boundary element 
1 analysis program Wadam [24]. Fig. 2 plots these hydrodynamic coefficients. It is worth noting that 2 although a linear wave force model has been employed to focus on the scope of the present research, 3 the developed dynamic model is able to simulate nonlinear dynamic effect. For example, a nonlinear 4 wave excitation force model $[25,26]$ or a nonlinear mooring module can be implemented.

\section{3. Control strategy}

\subsection{Complex-conjugate control}

As extensively presented in [3], the optimum condition for wave power extraction in regular wave 10 is

$$
u(\omega)=H(\omega) \cdot F_{\text {wave }}(\omega), H(\omega)=\frac{1}{2 \operatorname{Re}\{Z(\omega)\}}
$$

or

$$
\dot{z}(t)=H(\omega) \cdot f_{\text {wave }}(t), H(\omega)=\frac{1}{2 \operatorname{Re}\{Z(\omega)\}}
$$

12 where $\omega$ is the oscillating frequency of the regular wave. $F_{\text {wave }}(\omega)$ is the Fourier transform of wave 13 excitation force $f_{\text {wave }}(t)$ and $u(\omega)$ is the Fourier transform of buoy velocity $\dot{z}(t)$

$$
F_{\text {wave }}(\omega)=\int_{-\infty}^{\infty} f_{\text {wave }}(t) e^{-i \omega t} \mathrm{~d} t
$$

$$
u(\omega)=\int_{-\infty}^{\infty} \dot{z}(t) e^{-i \omega t} \mathrm{~d} t
$$

$15 Z(\omega)$, which is plotted in Fig. 3, is widely known as the intrinsic impedance. 


$$
Z(\omega)=\{\lambda(\omega)+D\}+i \cdot\left\{\omega[M+\mu(\omega)]-\frac{\rho g \pi R^{2}}{\omega}\right\}
$$

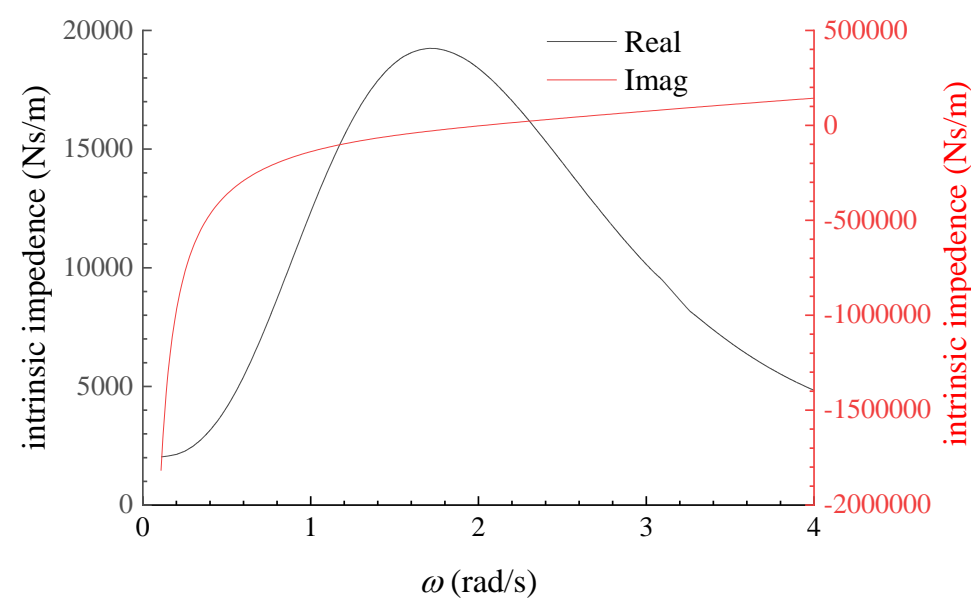

Fig. 3. Intrinsic impedance of the buoy.

Eq. (6) is the law of the classic optimal complex-conjugate control, which indicates that the optimum velocity is proportional to the wave force. Since the transfer function $H(\omega)$ is a real value, the optimum velocity is also in phase with the wave excitation force.

\subsection{Sub-optimal causal control}

By controlling the buoy velocity according to Eq. (6), which is a frequency-dependent constant gain, the power extraction of a WEC could be maximized. It is not a problem for regular wave analysis as $H(\omega)$ is a real value so that it is causal. In random waves, the optimum condition must be satisfied for all frequency components, resulting in an impulse response function $h(t)$, which is the inverse Fourier transform of the frequency domain transfer function $H(\omega)$

$$
\dot{z}(t)=\int_{-\infty}^{\infty} h(t-\tau) \cdot f_{\text {wave }}(\tau) \mathrm{d} \tau
$$

$$
h(t)=\frac{1}{2 \pi} \int_{-\infty}^{\infty} H(\omega) \cdot e^{i \omega t} \mathrm{~d} \omega=\frac{1}{\pi} \int_{0}^{\infty} H(\omega) \cdot \cos (\omega t) \mathrm{d} \omega(H(\omega) \text { is even })
$$

Eq. (9) is the time domain form of the complex-conjugate control law obtained through the Inverse Fourier transform. As shown in Fig. 4, $h(t)$ is non-zero for $t<0$ so that the transfer function is noncausal in the time domain as the current optimum velocity is dependent on future wave forces. If the future wave forces are fully known, the complex-conjugate control can be implemented successfully. However, the ocean waves are stochastic, and the coming wave forces must be predicted using a specific approach. Although various wave forecasting methods have been developed [12, 15], the forecasting error is still significant, which has been proved to have a negative influence on the control efficiency $[17,27]$. 

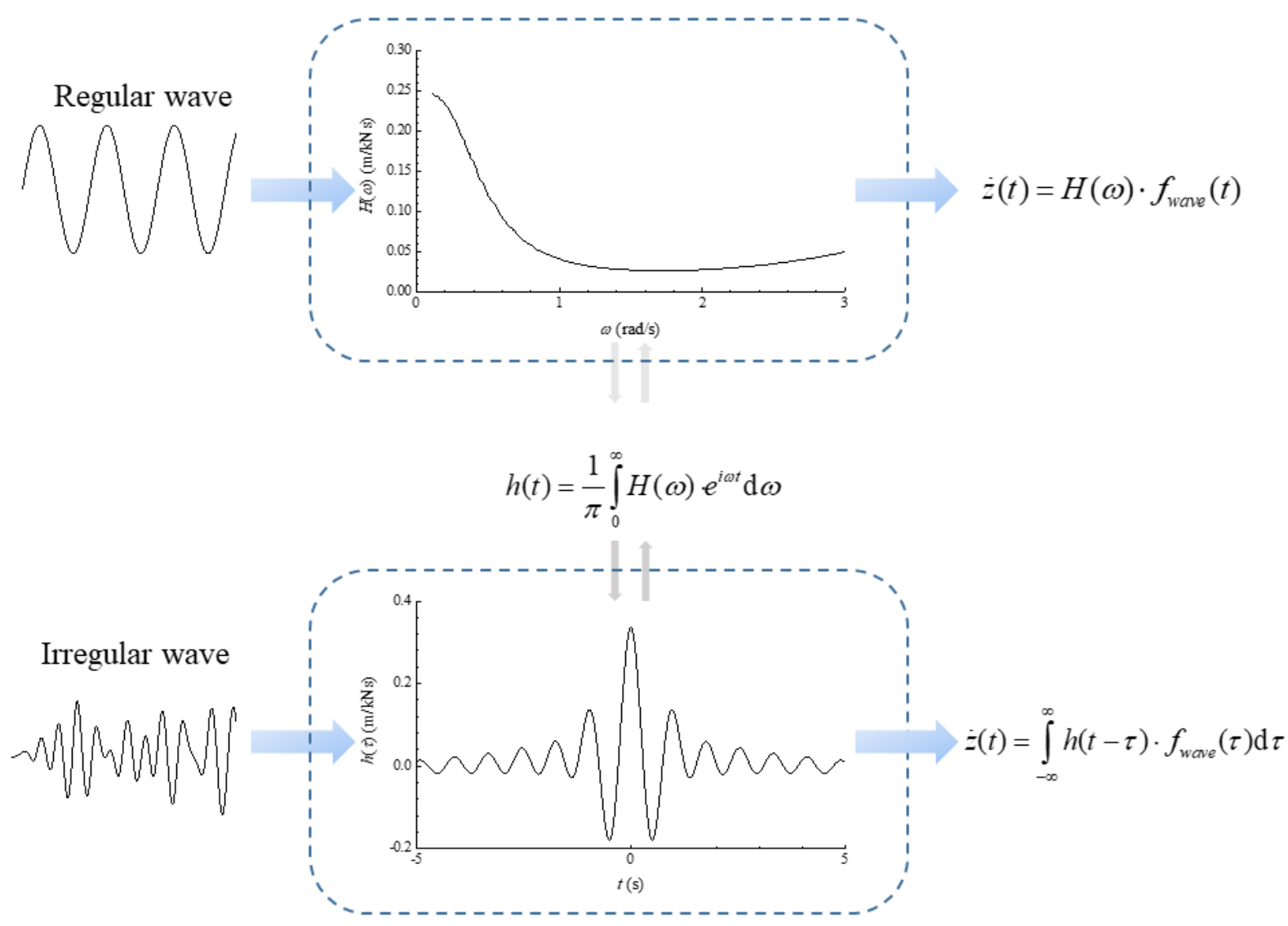

Fig. 4. Transfer function of the hemisphere buoy.

If the non-causal transfer function $h(t)$ is substituted by an approximation transfer function $\widehat{H}$, the complex-conjugate control becomes causal. In that case, Eq. (9) transforms to

$$
\dot{z}(t)=\hat{H} \cdot f_{\text {wave }}(t)
$$

Eq. (10) indicates that the non-causality can be removed via the introduction of an approximation transfer function since the future wave forces are no longer needed to derive the optimum velocity. A similar idea was documented previously [18], where the random wave force was assumed monochromatic so that the frequency domain transfer function for one single frequency (the wave peak frequency) can be utilized as the approximation transfer function. Such an assumption inherently indicates that the power extraction is just maximized at the wave peak period. However, the realistic sea state is stochastic, and the waves consist of multiple frequency components. Satisfying the optimum condition for the wave peak period alone cannot guarantee that the power extraction is maximized over the spectrum.

\subsection{Optimization of the causal transfer function}

16 The present research proposes an analytical model to optimize the approximation transfer function

$17 \widehat{H}$, which does not satisfy the optimum condition for one single frequency, but approaches to the 
optimum condition for each frequency based on a weight factor. Please note that the control algorithm is still sub-optimal even if the transfer function $\widehat{H}$ is optimized.

According to Falnes [3], the energy extraction is

$$
E=\int_{-\infty}^{\infty}-f_{P T O}(t) \dot{z}(t) \mathrm{d} t=\frac{1}{2 \pi} \int_{0}^{\infty}\left\{-F_{P T O}(\omega) u^{*}(\omega)-F_{P T O}^{*}(\omega) u(\omega)\right\} \mathrm{d} \omega
$$

where $F_{P T O}(\omega)$ is the Fourier transform of the PTO system mechanical force. * represents the complex conjugate. By algebraic manipulation, Eq. (11) is re-expressed

$$
E=\frac{1}{2 \pi} \int_{0}^{\infty} \frac{\left|F_{\text {wave }}(\omega)\right|^{2}}{2 \operatorname{Re}\{Z(\omega)\}} \mathrm{d} \omega-\frac{1}{2 \pi} \int_{0}^{\infty} \frac{F_{\text {wave }}(\omega) F_{\text {wave }}^{*}(\omega)+2 \operatorname{Re}\{Z(\omega)\}\left[F_{P T O}(\omega) u^{*}(\omega)+F_{P T O}^{*}(\omega) u(\omega)\right]}{2 \operatorname{Re}\{Z(\omega)\}} \mathrm{d} \omega
$$

Please note that $Z(\omega) \cdot u(\omega)=F_{\text {wave }}(\omega)+F_{P T O}(\omega)$. Insert it into Eq. (12)

$$
\begin{aligned}
E & =\frac{1}{2 \pi} \int_{0}^{\infty} \frac{\left|F_{\text {wave }}\right|^{2}}{2 \operatorname{Re}\{Z\}} \mathrm{d} \omega-\frac{1}{2 \pi} \int_{0}^{\infty} \frac{F_{\text {wave }} F_{\text {wave }}^{*}+2 \operatorname{Re}\{Z\}\left[F_{P T O} u^{*}+F_{P T O}^{*} u\right]}{2 \operatorname{Re}\{Z\}(\omega)} \mathrm{d} \omega \\
& =\frac{1}{2 \pi} \int_{0}^{\infty} \frac{\left|F_{\text {wave }}\right|^{2}}{2 \operatorname{Re}\{Z\}} \mathrm{d} \omega-\frac{1}{2 \pi} \int_{0}^{\infty} \frac{F_{\text {wave }} F_{\text {wave }}^{*}+2 \operatorname{Re}\{Z\}\left[Z u u^{*}-F_{\text {wave }} u^{*}+Z^{*} u u^{*}-F_{\text {wave }}^{*} u\right]}{2 \operatorname{Re}\{Z\}} \mathrm{d} \omega \\
& =\frac{1}{2 \pi} \int_{0}^{\infty} \frac{\left|F_{\text {wave }}\right|^{2}}{2 \operatorname{Re}\{Z\}} \mathrm{d} \omega-\frac{1}{2 \pi} \int_{0}^{\infty} \frac{\left|F_{\text {wave }}-2 \operatorname{Re}\{Z\} \cdot u\right|^{2}}{2 \operatorname{Re}\{Z\}} \mathrm{d} \omega
\end{aligned}
$$

The first term presents the maximum energy extraction whilst the second term is the energy loss. If the optimum condition Eq. (6) is satisfied for every frequency component, the energy loss is zero. As interpreted previously, it will lead to the non-causality in the real-time implementation of the complexconjugate control. Instead of eliminating the energy loss, the present research aims to find an approximation transfer function $\widehat{H}=u / F_{\text {wave }}$ that minimizes $\beta$ as much as possible

$$
\hat{H}=\min (\beta)
$$

$$
\beta=\frac{1}{2 \pi} \int_{0}^{\infty} \frac{\left|(1-2 \hat{H} \cdot \operatorname{Re}\{Z(\omega)\}) F_{\text {wave }}(\omega)\right|^{2}}{2 \operatorname{Re}\{Z(\omega)\}} \mathrm{d} \omega
$$

Please note that $\widehat{H}$ just minimizes $\beta$ but can't reduce it to zero as it can't satisfy the optimum condition for every frequency. Therefore, the proposed control is sub-optimal. Given the local wave spectrum $S(\omega)$

$$
F_{\text {wave }}(\omega)=e^{i W(\omega)} \cdot \psi(\omega) \sqrt{\pi S(\omega)}
$$

where $W(\omega)$ is a random value between 0 and $2 \pi . e^{i W(\omega)}$ represents the random phase of each wave component. Please note that $\left|e^{i W(\omega)}\right|=1 . \Psi(\omega)$ is the wave force response amplitude operator (RAO). Combing Eq. (15) and Eq. (14)

$$
\hat{H}=\min (\beta)
$$

$$
\beta=\frac{1}{2 \pi} \int_{0}^{\infty} \frac{|(1-2 \hat{H} \cdot \operatorname{Re}\{Z(\omega)\}) \psi(\omega) \sqrt{\pi S(\omega)}|^{2}}{2 \operatorname{Re}\{Z(\omega)\}} \mathrm{d} \omega
$$


For a given buoy, $\Psi(\omega)$ and $Z(\omega)$ are already determined so that Eq. (16) minimizes the energy loss

2 with consideration of the power density distribution of the wave spectrum. The optimum approximation

3 transfer function is the one that minimizes $\beta$. It is easy to find that $\beta$ reaches it minima at $\partial \beta / \partial \widehat{H}=0$.

4 Therefore, the optimum approximation transfer function is

$$
\hat{H}=\frac{\int_{0}^{\infty} \psi^{2}(\omega) S(\omega) \mathrm{d} \omega}{\int_{0}^{\infty} 2 \operatorname{Re}\{Z(\omega)\} \psi^{2}(\omega) S(\omega) \mathrm{d} \omega}
$$

The approximation transfer function has a similar pattern with the frequency-dependent optimal transfer function. As mentioned before, the approximation transfer function does not satisfy the optimum condition for one single frequency. Instead, it is designed to approach the optimum condition for each frequency with a weight factor. Although the specific form of this weight factor is not clear from Eq. (17), one can easily find that

$$
\frac{1}{\hat{H}}=\frac{\int_{0}^{\infty} 2 \operatorname{Re}\{Z(\omega)\} \psi^{2}(\omega) S(\omega) \mathrm{d} \omega}{\int_{0}^{\infty} \psi^{2}(\omega) S(\omega) \mathrm{d} \omega}
$$

$$
\approx \frac{\sum_{i=1}^{N} \frac{1}{H\left(\omega_{i}\right)} \psi^{2}\left(\omega_{i}\right) S\left(\omega_{i}\right) \Delta \omega_{i}}{\sum_{j=1}^{N} \psi^{2}\left(\omega_{j}\right) S\left(\omega_{j}\right) \Delta \omega_{j}}
$$

It indicates that the reciprocal of the approximation transfer function is the weighted sum of that of the frequency-dependent optimal transfer function, and the weight factor $\varepsilon$ is

$$
\varepsilon\left(\omega_{i}\right)=\frac{\psi^{2}\left(\omega_{i}\right) S\left(\omega_{i}\right) \Delta \omega_{i}}{\sum_{j=1}^{N} \psi^{2}\left(\omega_{j}\right) S\left(\omega_{j}\right) \Delta \omega_{j}}
$$

According to Eq. (18), the frequency-dependent transfer function is weighted based on the power density distribution of the wave spectrum. If $N=1$, the approximation transfer function is exactly the optimal frequency-dependent one. It indicates that the proposed control is identical to the classic complex-conjugate control in the monochromatic wave.

Fig. 5 compares the optimal transfer function, the approximation transfer function derived by Eq. (17), and the approximation transfer function estimated based on wave peak period. One can see that the proposed control strategy employs a different approximation transfer function, which will lead to discrepancies on the power extraction. 


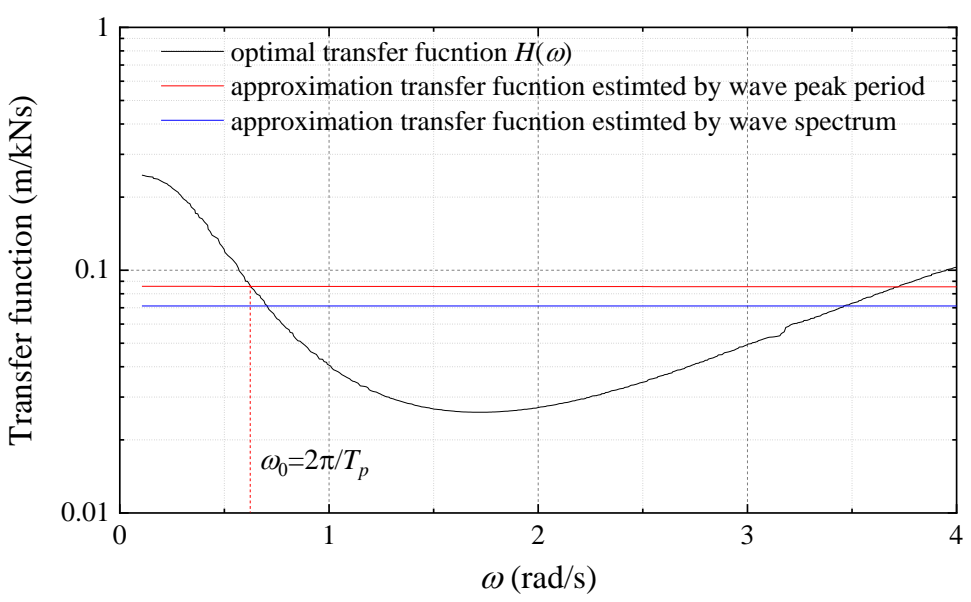

Fig. 5 Bode magnitude of the transfer function $(H s=4 \mathrm{~m}, T p=10 \mathrm{~s})$.

Fig. 6 plots how $\beta$ varies with the approximation transfer function. The dots represent the approximation transfer function based on the peak period of the spectrum. According to Fig. 6, if the approximation transfer function is just selected based on peak period and neglects the power distribution of the wave spectrum, $\beta$ is not minimized sufficiently, especially when the wave peak period is relatively long. Hereinafter, Eq. (17) will be used to derive the optimum approximation transfer function. Please note that the present research does not consider any physical constraint, e.g. the upper bound of realtime power extraction and maximum displacement.

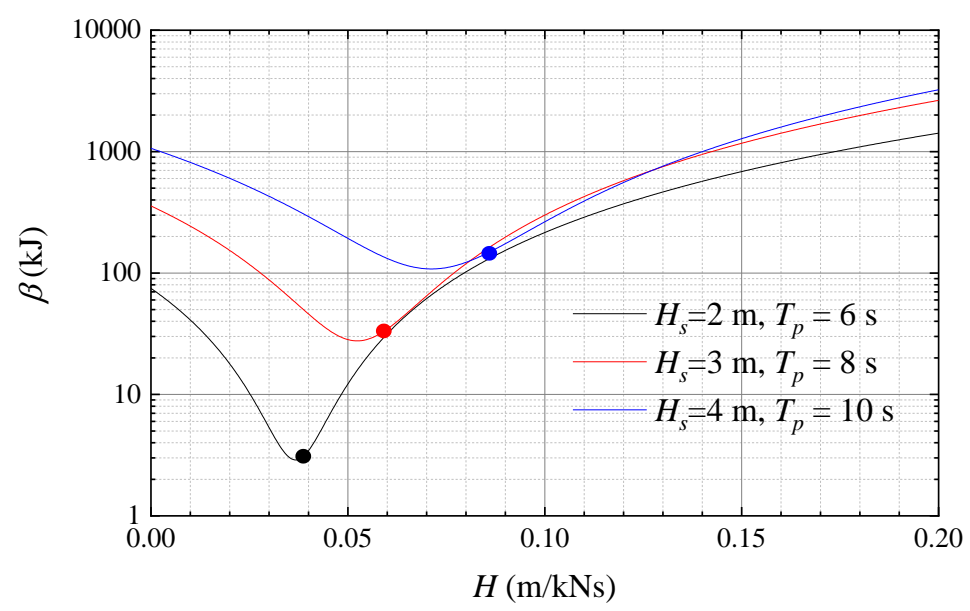

Fig. 6. Power loss with the approximation transfer function.

\subsection{Velocity-tracking}

By substituting the time domain non-causal transfer function Eq. (9) with the approximation transfer function Eq. (17), the non-causality of wave energy control is removed so that the future wave forces are no more required. Based on the approximation transfer function, the desired buoy velocity is obtained, and the power extraction could be increased by controlling the real-time buoy velocity via tuning the PTO mechanical force. In the present research, the PID control is used to tune the PTO force. Fig. 7 illustrates how the WEC velocity is controlled based on the optimized approximation transfer function. Please note that the wave force is difficult to measure in practice and usually, it is estimated 
1 based on the buoy motion using a specially developed model [28]. To focus on the scope of the present 2 research, it is assumed that the wave force is measurable.

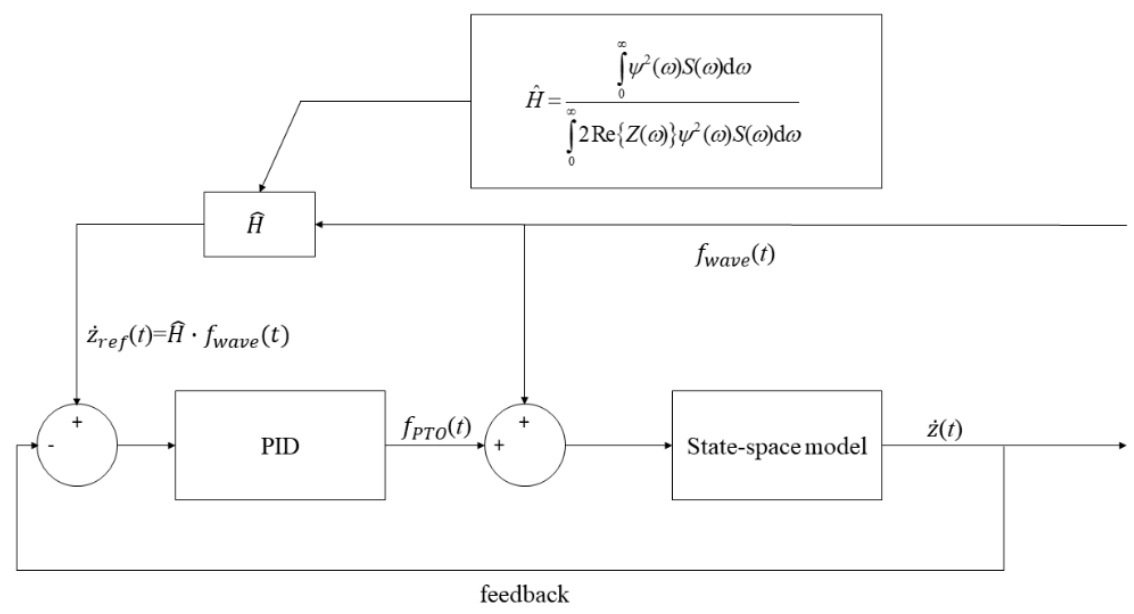

3

\section{4. Validation}

\subsection{WEC dynamic model}

Firstly, the dynamic model of the WEC is cross-verified against hydrodynamic analysis tool Wadam

8 [24]. The buoy velocity in the absence of the PTO mechanical force under a set of regular waves 9 estimated by Wadam and the present dynamic model is compared in Fig. 8. The simulations run until steady state is reached. As shown, the agreement between the two simulation tools is good.

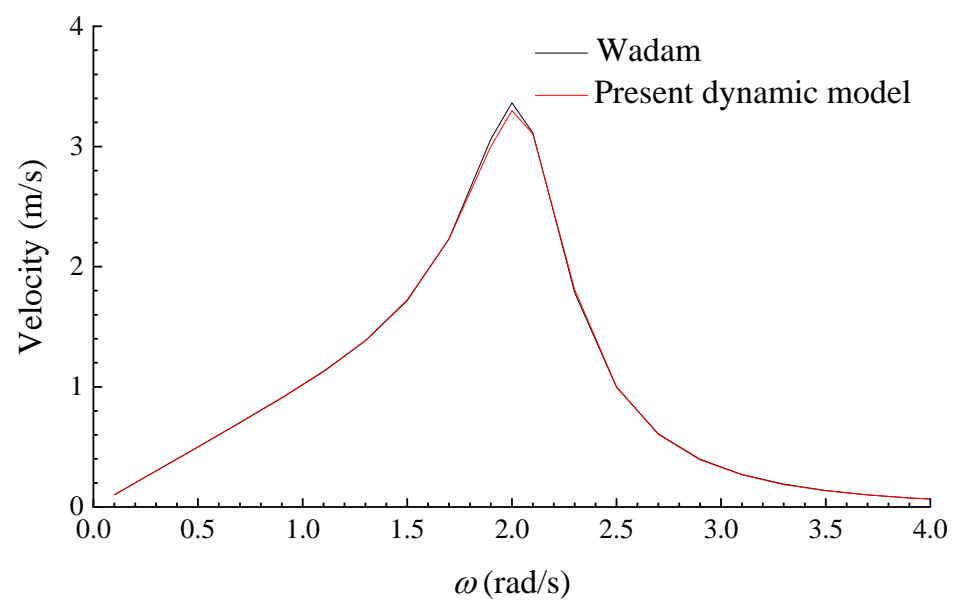

Fig. 8. Buoy velocity induced by unit amplitude regular waves

\subsection{Complex-conjugate control}

14 According to the above discussions, the present causal control algorithm is an approximation of the 15 complex-conjugate in random waves and reduces to the complex-conjugate control in regular waves. 16 Nebel [10] gave the optimal power extraction 


$$
\begin{aligned}
& \left.\frac{1}{2} \sum_{i=1}^{N}\left[\operatorname{Re}\left\{F\left(\omega_{i}\right)\right\} \cdot \operatorname{Re}\left\{\text { 诲 } \omega_{i}\right)\right\}+\operatorname{Im}\left\{\hat{F}\left(\omega_{i}\right)\right\} \cdot \operatorname{Im}\left\{v\left(\omega_{i}\right)\right\}\right] \\
& \hat{v}\left(\omega_{i}\right)=\frac{1}{2 \operatorname{Re}\left\{Z\left(\omega_{i}\right)\right\}} \cdot f_{\text {wave }}\left(\omega_{i}\right) \\
& \hat{F}\left(\omega_{i}\right)=\frac{Z^{*}\left(\omega_{i}\right)}{2 \operatorname{Re}\left\{Z\left(\omega_{i}\right)\right\}} \cdot f_{\text {wave }}\left(\omega_{i}\right)
\end{aligned}
$$

\section{5. Simulation results}

where $f_{\text {wave }}(\omega)$ is the wave force. The present control algorithm is validated against the theoretical optimal power extraction given by Eq. (20). Fig. 9 compares the power extraction with the current control algorithm based on PID tuning and the theoretical limit. The theoretical maximum power extraction is generally obtained with the present PID controller.

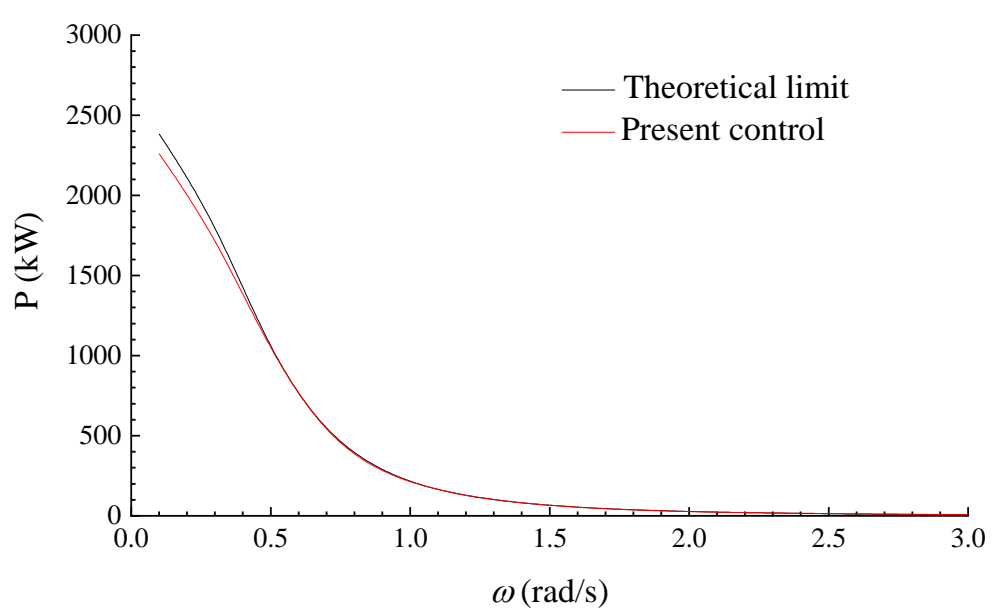

Fig. 9. Optimal energy extraction in unit amplitude regular waves.

In this section, the efficiency and the robustness of the proposed control strategy in a wide range of wave conditions are examined. The random waves are specified by the JONSWAP wave spectrum. The wave parameters considered are listed in Table 1. In each load case, the approximation transfer function is first obtained based on the wave spectrum selected using Eq. (17). Afterwards, the desired velocity is tuned with the PID control illustrated in Fig. 7.

In real conditions, the local metocean information, e.g. monthly average wave condition, are usually investigated before a WEC is installed. Then the approximation transfer function is determined in advance and employed using the look-up-table approach. The switch between different $\widehat{H}$ or monthly sea state can be achieved through an electronic time meter, which will give a command to update $\widehat{H}$ every month. A more efficient way is to utilize the wave forecasting released very few hours in advance by national weather centres, e.g., UK Met Office, to update the approximation transfer function. This approach has two primary advantages. First of all, the sea state is updated much more frequently and thus $\widehat{H}$ can be tuned in a quasi-real-time manner. Also, the information offered by weather centres using numerical models which are calibrated against measurements using advanced techniques, e.g., remote 
1 sense, are more precise than the monthly wave data. The accuracy of wave forecasting for 6-12 hours

2 ahead is quite good for the application here. It ensures that the approximation transfer function matches

3 the actual local sea state. Due to the two advantages, the control efficiency would be further improved

4 if the forecasted wave condition is employed.

5 Table 1 Wave conditions

\begin{tabular}{lllll}
\hline & $H_{s}(\mathrm{~m})$ & $T_{p}(\mathrm{~s})$ & $\gamma$ & $\widehat{H}(\mathrm{~m} / \mathrm{kNs})$ \\
\hline Env1 & 2 & 6 & 3 & $3.68 \times 10^{-2}$ \\
Env2 & 3 & 8 & 3 & $5.24 \times 10^{-2}$ \\
Env3 & 4 & 10 & 3 & $7.12 \times 10^{-2}$ \\
\hline
\end{tabular}

6

7 5.1. Enhancement of power extraction

8 First, the improvement of power extraction due to the proposed non-causal control is investigated.

9 In the absence of the control, the PTO force is commonly simplified as a linear damping coefficient,

10 which is a reasonable approximation of the direct generator PTO system [29]. In this circumstance, the

11 PTO force is $p_{\text {ot }}=-D_{\text {PTO }} \dot{z}$. Fig. 10 demonstrates the sensitivity of the power extraction to wave

12 frequency, which is based on a set of regular waves with unit wave amplitude $(1 \mathrm{~m})$. As shown, the 13 optimal PTO damping coefficient is dependent on the wave frequency.

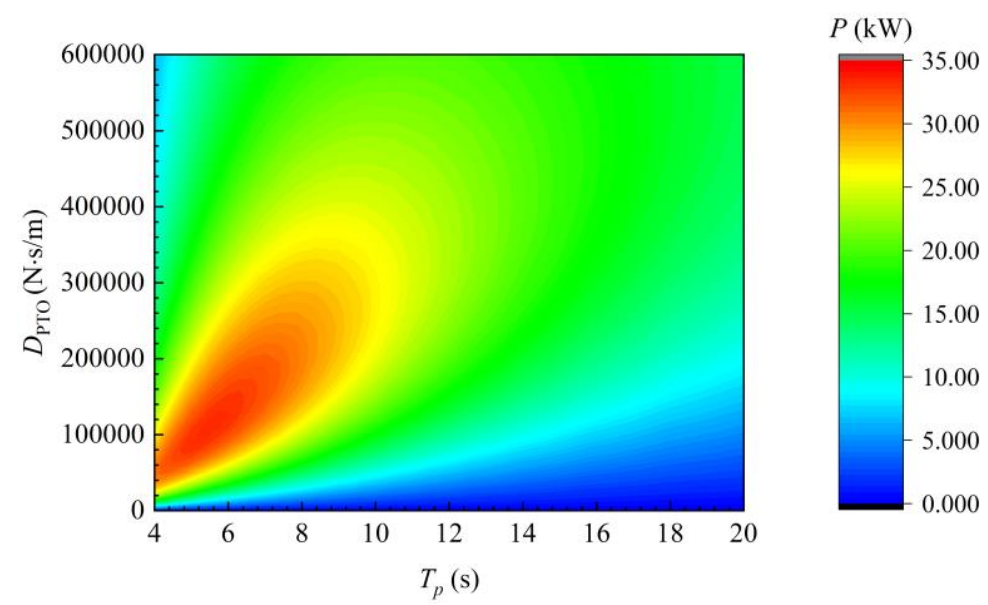

Fig. 10. Sensitivity of power extraction to PTO damping coefficient.

Fig. 11 plots the energy converted during 1 hour period, which clearly shows that the wave energy conversion accumulates more rapidly when the proposed causal control strategy is implemented. It is with noting that the application of complex conjugate control requires the PTO force to return some energy during part of the oscillation cycle, leading to the fluctuations observed from the power accumulation. Table 2 lists the power extraction of the WEC with and without the proposed control. It is shown that the power extraction increases substantially with the implementation of the proposed control algorithm. 
Table 2 Power extraction with and without control

\begin{tabular}{llll}
\hline & Linear damping $(\mathrm{kW})$ & $D_{\text {PTO }}(\mathrm{kNs} / \mathrm{m})$ & With control $(\mathrm{kW})$ \\
\hline Env1 & 7.2 & 130 & 68.2 \\
Env2 & 15.5 & 200 & 294.5 \\
Env3 & 23.1 & 260 & 831.5 \\
\hline
\end{tabular}

Fig. 11 Total energy converted during 1 hour period, Env1

The control performance is further assessed in term of relative capture width

$$
R C W=\frac{P}{2 R \cdot P_{\text {wave }}}
$$

where $P_{\text {wave }}=\rho \mathrm{g}^{2} H_{s}^{2} T_{p} /(64 \pi)$ is the average wave power per meter of wave front.

Table 3 compares the power extraction using the proposed causal control and the theoretical maximum power extraction. Generally, $80 \%-90 \%$ of the theoretical maximum power extraction could be achieved with the present causal control strategy. The capture width of the proposed causal control is not narrowed much. As illustrated in Section 3.2, the non-causality of the complex-conjugate control is eliminated by replacing the non-causal optimal transfer function with the approximation one. The approximation transfer function does not satisfy the optimum condition for all frequency components, and the energy loss is not eliminated. Therefore, the proposed causal control algorithm is sub-optimal. Even so, the non-causality is removed and the control efficiency is generally satisfactory.

Table 3 Efficiency of the proposed control.

\begin{tabular}{ccccc}
\hline & \multicolumn{2}{c}{ Sub-optimal causal control } & \multicolumn{2}{c}{ Optimal non-causal control } \\
\hline & $P(\mathrm{~kW})$ & $C W(\mathrm{~m})$ & $\widehat{P}(\mathrm{~kW})$ & $\widehat{C W}(\mathrm{~m})$ \\
\hline Env1 & 68.2 & 5.80 & 74.7 & 6.36 \\
Env2 & 294.5 & 8.35 & 356.5 & 10.11 \\
Env3 & 831.5 & 10.61 & 1065.1 & 13.60 \\
\hline
\end{tabular}

A general conclusion from Table 3 is that the proposed causal control is less efficient in long peak period wave condition. The reduction of efficiency indicates that the approximation transfer function Eq. (17) is a worse approximation of the frequency-dependent optimal transfer function in the presence of long waves. To further interpret the strategy behind, the wave spectrums and the frequencydependent transfer function are plotted together in Fig. 12. In Env1, the majority of wave power is

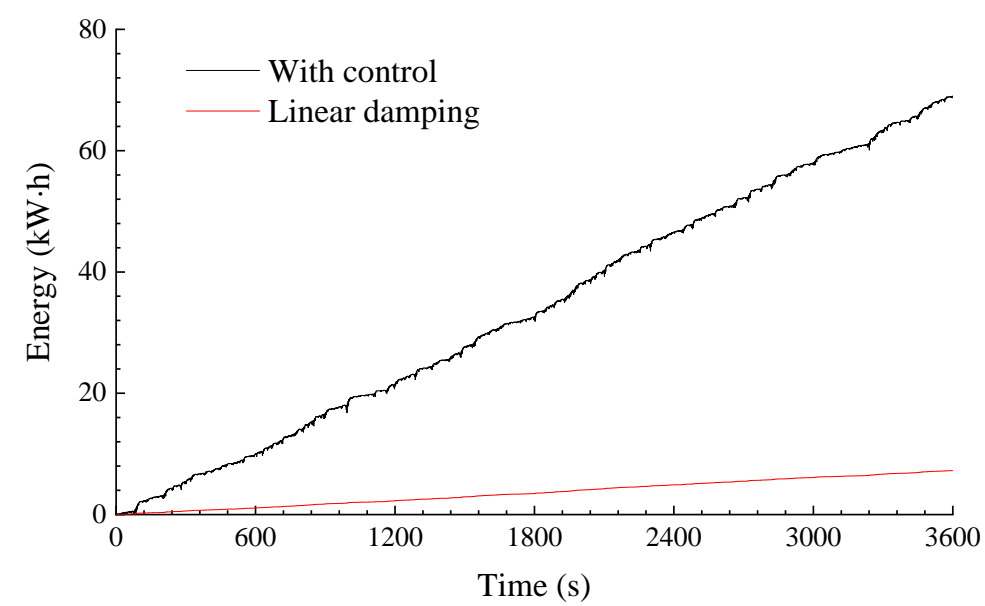


1 distributed around 4-8 s. Please note the transfer function does not vary much within this range. It 2 indicates that it is possible to approximate the optimal frequency-dependent transfer function $H(\omega)$ 3 using $\widehat{H}$. Actually, the proposed control strategy is optimal if the frequency-dependent transfer function 4 is constant. When the peak period of the random waves increases, the wave spectrum shifts to the long5 period region and the wave spectrum bandwidth is broadened. It can be seen that the transfer function 6 varies a lot in Env3. Therefore, $\widehat{H}$ is a worse approximation of the optimal frequency-dependent transfer 7 function $H(\omega)$.

\section{5.2. Sensitivity of the power extraction} is investigated through the tuning of damping coefficient $D$.

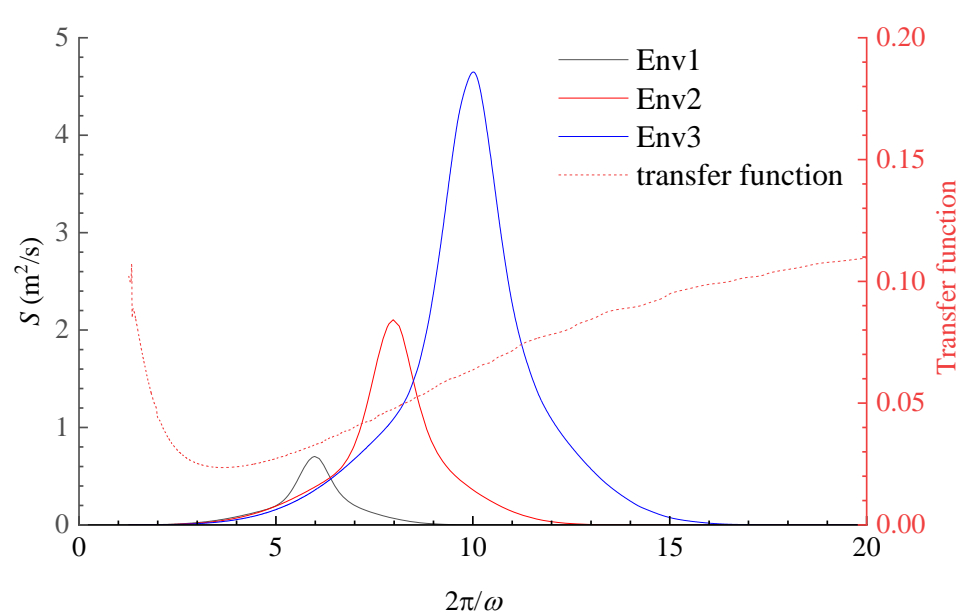

Fig. 12. Wave spectrum $S(\omega)$ and frequency domain transfer function $H(\omega)$.

This section examines the sensitivity of wave power extraction to the power density distribution of the wave spectrum to the viscous effect. Load case Env3 is considered here. The power density distribution is modified by adjusting the peak parameter manually. The sensitivity to the viscous force

The variation of power extraction with viscous wave force is demonstrated in Fig. 13 and Table 4. Both the actual power extraction and the theoretical limit decreases with the viscous wave force, which suppresses WEC dynamic response. It is interesting to find that the proposed control algorithm is more efficient in the presence of high-level viscous wave force. When the additional damping coefficient increases from $1 \%$ critical damping to $3 \%$ critical damping, the control efficiency is increased by $10 \%$. 


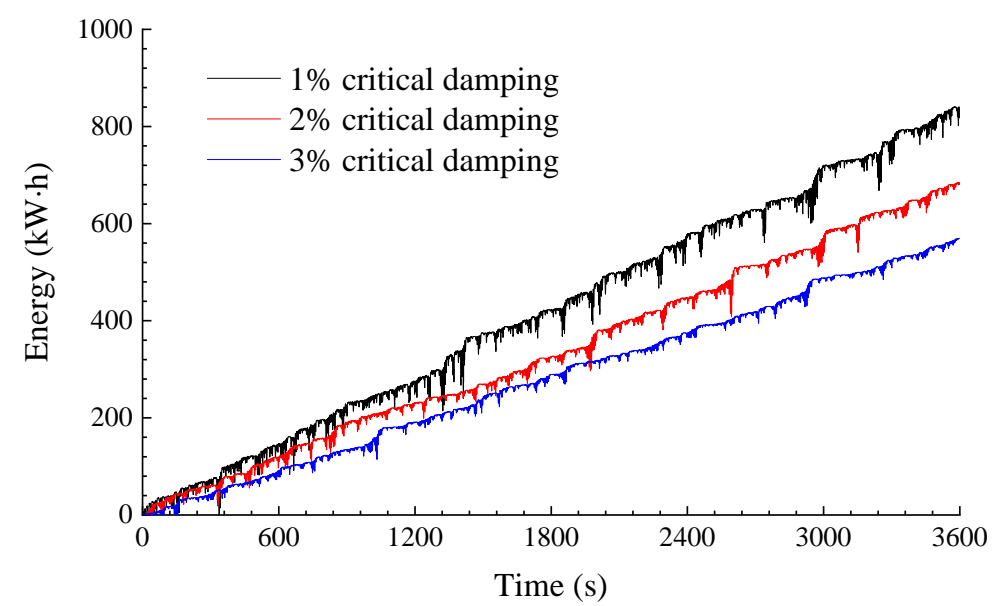

Fig. 13 Effect of viscous force on total energy absorption.

Table 4 Sensitivity of energy extraction to viscous wave force (Env3)

\begin{tabular}{ccccc}
\hline & \multicolumn{2}{c}{ Sub-optimal causal control } & \multicolumn{2}{c}{ Optimal non-causal control } \\
\cline { 2 - 5 } & $P(\mathrm{~kW})$ & $R C W$ & $\widehat{P}(\mathrm{~kW})$ & $\widehat{R C W}$ \\
\hline 1\% critical damping & 831.5 & 2.12 & 1065.1 & 2.72 \\
$2 \%$ critical damping & 671.7 & 1.71 & 797.0 & 2.03 \\
$3 \%$ critical damping & 560.5 & 1.43 & 639.7 & 1.63 \\
\hline
\end{tabular}

4

5

6

7

8

9

According to Section 3.3, it is easy to find that the efficiency of the proposed control strategy is dependent on the pattern of the transfer function. The approximation transfer function is a good approximation if the transfer function is relatively flat. In the ideal case, when the transfer function is entirely flat and constant, the approximation transfer function is just the frequency-dependent transfer function. Fig. 14 plots the transfer function with different levels of viscous wave force. As shown, the optimal transfer function $H(\omega)$ becomes flatter as the level of viscosity increases so that the approximation transfer function is a better approximation of $H(\omega)$ and thereby the control efficiency $\eta$ is higher.

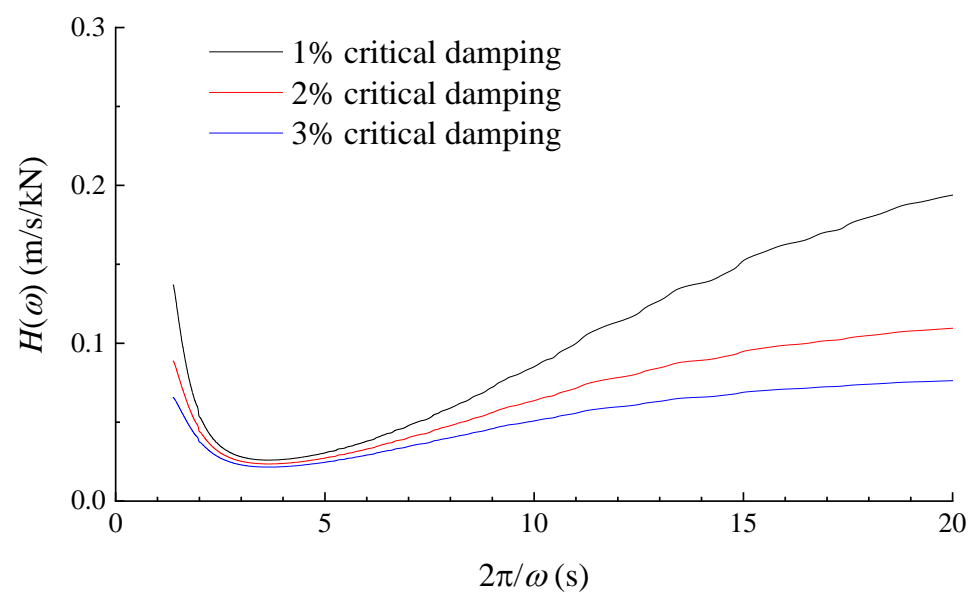

Fig. 14. Transfer function with different levels of viscosity.

Fig. 15 plots the wave spectrums with different peak parameters. As shown, a large peak parameter represents a narrow-banded spectrum. Table 5 illustrates how the power density distribution of the wave 
1 spectrum influences the control performance. Generally, the proposed control strategy is less efficient 2 in broad-banded waves, and the explanation has been given previously. Nevertheless, the efficiency 3 does not reduce much. It indicates that the proposed causal control will also be useful in broad-banded 4 waves.

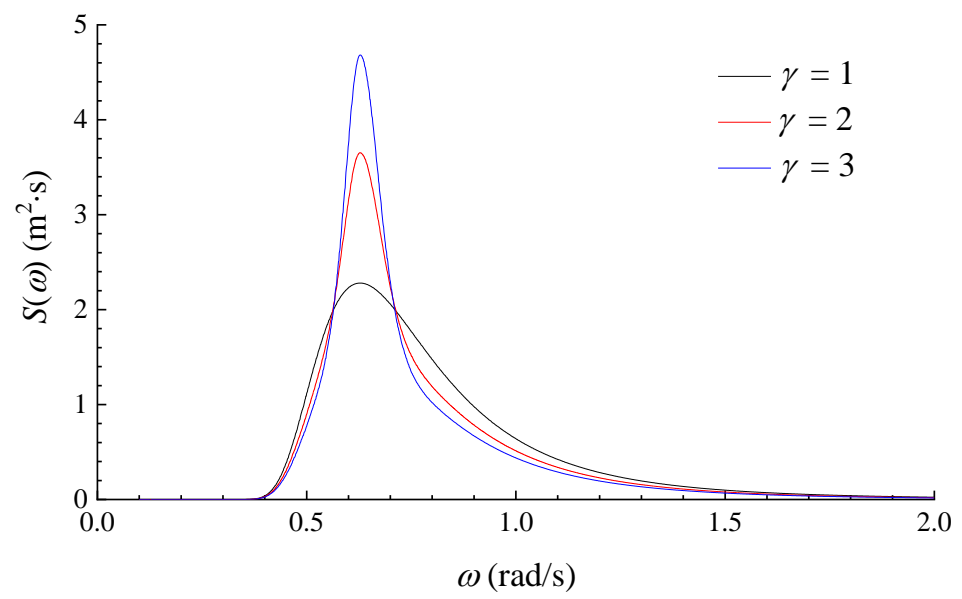

Fig. 15. Wave spectrum with arbitrary shape factors, Env3.

Table 5 Sensitivity of energy absorption to wave spectrum shape $\left(D=0.01 \times 2 \sqrt{\rho g \pi R^{2}(M+m)}\right.$, Env3)

\begin{tabular}{ccccc}
\hline & \multicolumn{2}{c}{ Sub-optimal causal control } & \multicolumn{2}{c}{ Optimal non-causal control } \\
\cline { 2 - 5 } & $P(\mathrm{~kW})$ & $R C W$ & $\widehat{P}(\mathrm{~kW})$ & $\widehat{R C W}(\mathrm{~m})$ \\
\hline$\gamma=1$ & 721.0 & 1.84 & 970.4 & 2.48 \\
$\gamma=2$ & 786.8 & 2.01 & 1025.9 & 2.62 \\
$\gamma=3$ & 831.5 & 2.12 & 1065.1 & 2.72 \\
\hline
\end{tabular}

8

According to the derivation of the approximation transfer function, the proposed control algorithm is based on the wave spectrum and therefore takes the power density distribution into account. It is the advantage of the present control algorithm against previous methods, which just satisfy the optimum condition at the wave peak period. Fig. 16 compares the control performance when different approximation transfer function models are utilized. Regardless of the wave spectrum bandwidth, the WEC extracts more wave energy if the approximation transfer function is derived based on Eq. (17). It indicates that if the transfer function at the wave peak period is selected as the approximation transfer function, the energy loss is not reduced sufficiently, which can be proved by Fig. 6 . The advantage of the proposed control becomes increasingly noticeable as the wave spectrum bandwidth is broadened. When the wave spectrum shape parameter is 1 , the WEC extracts $15 \%$ more wave power with the proposed control strategy. As shown, the efficiency of control based on wave peak period reduces to $59 \%$ in load case $\gamma=1$. It is why previous works mostly assumed that the sea waves are narrow-banded. 


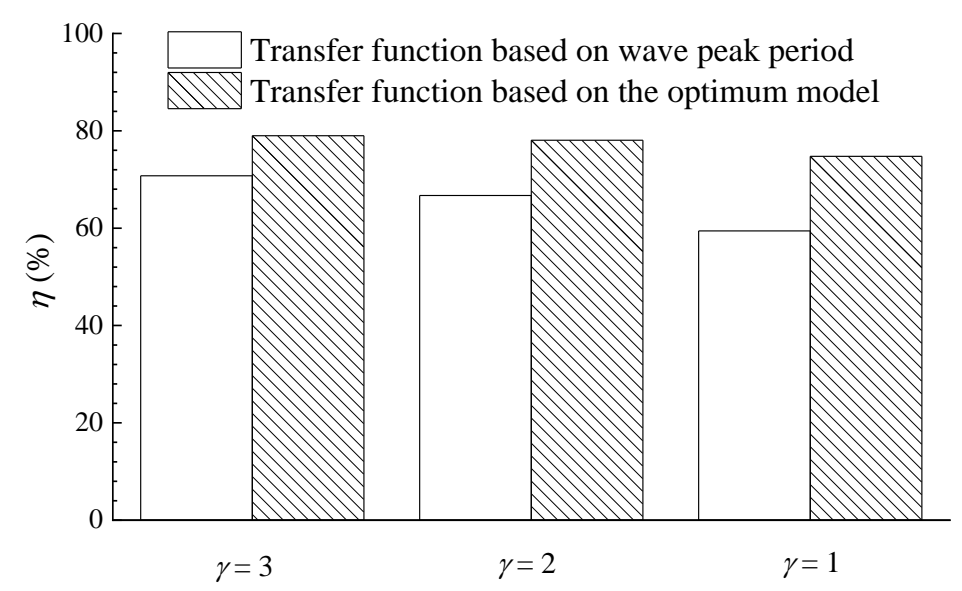

Fig. 16. Sensitivity of energy absorption to spectrum shape, $\eta=P / \widehat{P}$ (Env3).

\section{6. Conclusions}

A sub-optimal causal control strategy is developed in the present research, which is an approximation of the complex-conjugate control. An approximation transfer function is used to tune the velocity amplitude based on the current wave excitation force so that the non-causality is eliminated. An analytical optimization model of the approximation transfer function, based on the power density distribution of the local wave spectrum, is proposed. The approximation transfer function approaches the optimum condition for each frequency component with a weight factor. Since the optimization model cannot satisfy the optimum power extraction condition at every frequency component, the proposed control is thus sub-optimal.

The numerical simulations show that the proposed causal control could extract $75 \%-90 \%$ of the theoretical maximum power extraction. Moreover, the control efficiency remains hardly reduced even when the wave spectrum is broadened. It indicates that the proposed control is robust to the wave conditions so that it can works effectively in a wide range of sea states.

The advantage of the present strategy against previous methods, which merely maximize the power extraction at the wave peak period, is also highlighted. The proposed control algorithm is able to extract more wave energy than previous methods. More importantly, the present method has better robustness to the bandwidth of the wave spectrum.

\section{Acknowledgment}

The present research is supported by the State Key Laboratory of Coastal and Offshore Engineering, Dalian University of Technology (Grant No. LP1908) and State Key Laboratory of Hydraulic Engineering Simulation and Safety, Tianjin University (Grant No. HESS-1919). The first author

24 is grateful for their financial supports. 


\section{References}

2 [1] Zhang XT, Tian XL, Xiao LF, Li X, Chen LF. Application of an adaptive bistable power capture 3 mechanism to a point absorber wave energy converter. Appl Energ. 2018;228:450-67.

4 [2] Li L, Zhang XT, Yuan ZM, Gao Y. Multi-Stable Mechanism of an Oscillating-Body Wave Energy 5 Converter. Ieee T Sustain Energ. 2020;11(1):500-8.

6 [3] Falnes J. Ocean waves and oscillating systems: linear interactions including wave-energy extraction: 7 Cambridge university press, 2002.

8 [4] Budal K, Falnes J. Interacting point absorbers with controlled motion, in Power from Sea Waves:

9 BM Count, Academic Press, 1980.

10 [5] Babarit A, Guglielmi M, Clement AH. Declutching control of a wave energy converter. Ocean Eng. $11 \quad 2009 ; 36(12-13): 1015-24$.

12 [6] Todalshaug JH, Ásgeirsson GS, Hjálmarsson E, Maillet J, Möller P, Pires P, et al. Tank testing of 13 an inherently phase-controlled wave energy converter. Int J Mar Energy. 2016;15:68-84.

14 [7] Mérigaud A, Ringwood JV. A nonlinear frequency-domain approach for numerical simulation of wave energy converters. Ieee T Sustain Energ. 2017;9(1):86-94.

16 [8] Faedo N, Scarciotti G, Astolfi A, Ringwood JV. Energy-maximising control of wave energy converters using a moment-domain representation. Control Engineering Practice. 2018;81:85-96.

[9] Williams P. Application of pseudospectral methods for receding horizon control. J Guid Control Dynam. 2004;27(2):310-4. [10] Nebel P. Maximizing the Efficiency of Wave-Energy Plant Using Complex-Conjugate Control. Proceedings of the Institution of Mechanical Engineers, Part I: Journal of Systems and Control Engineering. 2016;206(4):225-36.

[11] Henriques JCC, Gato LMC, Falcao AFO, Robles E, Fay FX. Latching control of a floating oscillating-water-column wave energy converter. Renew Energ. 2016;90:229-41.

[12] Fusco F, Ringwood JV. Short-Term Wave Forecasting for Real-Time Control of Wave Energy Converters. Ieee T Sustain Energ. 2010;1(2):99-106.

[13] Li L, Yuan ZM, Gao Y. Maximization of energy absorption for a wave energy converter using the deep machine learning. Energy. 2018;165:340-9. [14] Wang H, Lei Z, Zhang X, Zhou B, Peng J. A review of deep learning for renewable energy forecasting. Energy Conversion and Management. 2019;198:111799. [15] Truong DQ, Ahn KK. Wave prediction based on a modified grey model $\operatorname{MGM}(1,1)$ for real-time control of wave energy converters in irregular waves. Renew Energ. 2012;43:242-55.

[16] Belmont MR, Horwood JMK, Thurley RWF, Baker J. Filters for linear sea-wave prediction. Ocean Eng. 2006;33(17-18):2332-51. Wave Energy Converter With Real-Time Control. Ieee T Sustain Energ. 2019;10(2):615-24. 
1 [18] Fusco F, Ringwood JV. Suboptimal Causal Reactive Control of Wave Energy Converters Using a

2 Second Order System Model. The Twenty-first International Offshore and Polar Engineering

3 Conference. Maui, Hawaii, USA: International Society of Offshore and Polar Engineers; 2011. p. 8.

4 [19] Shek JKH, Macpherson DE, Mueller MA, Xiang J. Reaction force control of a linear electrical 5 generator for direct drive wave energy conversion. Iet Renew Power Gen. 2007;1(1):17-24.

6 [20] Wu F, Zhang XP, Ju P, Sterling MJH. Optimal Control for AWS-Based Wave Energy Conversion 7 System. Ieee T Power Syst. 2009;24(4):1747-55.

8 [21] Cummins W. The impulse response function and ship motions. Washington DC: David Taylor 9 Model Basin; 1962.

10 [22] Perez T, Fossen TI. Practical aspects of frequency-domain identification of dynamic models of 11 marine structures from hydrodynamic data. Ocean Eng. 2011;38(2):426-35.

12 [23] Penalba M, Ringwood JV. A high-fidelity wave-to-wire model for wave energy converters. Renew 13 Energ. 2019;134:367-78.

14 [24] Veritas DN. WADAM-Wave Analysis by Diffraction and Morison Theory. SESAM user's 15 manual, Høvik1994.

16 [25] Penalba M, Mérigaud A, Gilloteaux J-C, Ringwood JV. Influence of nonlinear Froude-Krylov 17 forces on the performance of two wave energy points absorbers. Journal of Ocean Engineering and 18 Marine Energy. 2017;3(3):209-20.

19 [26] Penalba M, Ringwood JV. Linearisation-based nonlinearity measures for wave-to-wire models in 20 wave energy. Ocean Eng. 2019;171:496-504.

21 [27] Zhang XT, Yang JM, Zhao WH, Xiao LF. Effects of wave excitation force prediction deviations 22 on the discrete control performance of an oscillating wave energy converter. Ships Offshore Struc. 23 2016;11(4):351-68.

24 [28] Li L, Gao Z, Yuan Z-M. On the sensitivity and uncertainty of wave energy conversion with an artificial neural-network-based controller. Ocean Eng. 2019;183:282-93. 